\title{
Talleres experimentales para contribuir a la enseñanza de las Ciencias Naturales en la escuela primaria
}

\author{
MAURO PORCEL DE PERALTA ${ }^{(1)}$ \\ MARÍA SILVINA REYES ${ }^{(2)}$ \\ HÉCTOR ODETTI (3)
}

Fecha de recepción: 14/04/2020

Fecha de aceptación: 31/07/2020

Resumen. El presente trabajo describe la realización de talleres de capacitación docente llevados a cabos en dos escuelas primarias de la provincia de Santa Fe, con el objetivo de divulgar el conocimiento científico-tecnológico a través de la realización de actividades prácticas, experiencias y demostraciones propias de disciplinas como la Química, la Física y la Biología. En cada encuentro se priorizó la difusión de estrategias, herramientas y técnicas innovadoras que permitieron actualizar contenidos estructurantes de las Ciencias Naturales, poniendo en valor el laboratorio móvil. Al finalizar los talleres se hicieron encuestas a las y los participantes para conocer sus apreciaciones. Los resultados fueron altamente satisfactorios valorando como muy buenas las actividades propuestas, pudiendo concluir que la incitativa resultó motivadora e innovadora, ya que facilitó diferentes estrategias didácticas para la enseñanza de las Ciencias Naturales en el nivel primario.

Palabras clave. educación primaria . talleres docentes $\cdot$ Ciencias Naturales $\cdot$ laboratorio
(1) Facultad de Biología y Ciencias Biológicas, Universidad Nacional del Litoral (FBCB/UNL). Licenciado en Biotecnología. Doctorando en Educación en Ciencias Experimentales. Becario de Agencia en la Cátedra de Química General e Inorgánica de la Facultad de Bioquímica y Ciencias Biológicas de la Universidad Nacional del Litoral (UNL). Docente de Nivel Medio. Miembro de proyectos de investigación de la UNL

mauropdp@yahoo.com.ar

(2) Facultad de Humanidades y Ciencias, Universidad Nacional del Litoral (FHUC/UNL). Licenciada en Biodiversidad, Magister en Ciencia y Tecnología de los Alimentos y Doctora en Educación en Ciencias Experimentales. Docente-Investigadora en la Cátedra de Química General e Inorgánica de la Facultad de Humanidades y Ciencias de la Universidad Nacional del Litoral (UNL). Miembro de proyectos de extensión e investigación en el marco de la UNL. mariasilvinareyes@hotmail.com

(3) Facultad de Biología y Ciencias Biológicas, Universidad Nacional del Litoral (FBCB/UNL). Bioquímico y Doctor en Ciencias Biológicas. Profesor Titular Exclusiva del Dpto. de Química General e Inorgánica de la FBCB/UNL. Categoría I del Programa de Incentivos. Director de la Maestría en Didáctica de las Ciencias Experimentales y del Doctorado en Educación en Cs Experimentales. Director del Programa de Enseñanza PreUniversitario de la Universidad Nacional del Litoral. hodetti@fbcb.unl.edu.ar 


\section{Experimental Workshops as a Contribution to Teaching Natural Sciences in Primary School}

\begin{abstract}
This work describes the development of teacher training workshops at two primary schools in the province of Santa Fe, Argentina, which were intended to spread scientific and technological knowledge through practical activities, experiences, and demonstrations of disciplines such as Chemistry, Physics, and Biology. In each meeting, priority was given to the demonstration of innovative strategies, tools, and techniques that enabled the updating of structuring contents of Natural Sciences, highlighting the value of a mobile laboratory. At the end
\end{abstract}

of the workshops, participants were surveyed to find out their appreciations. Results were highly satisfactory, the proposed activities being assessed as very good. This allowed us to conclude that the initiative was motivating and innovative, since it promoted various didactic strategies for teaching Natural Sciences at primary level.

Keywords. primary education - teaching workshops . Natural Sciences $\cdot$ laboratory

\section{Introducción}

En la actualidad, tanto la sociedad en general como el sistema educativo en particular, muestran interés en identificar qué aprenden y qué deberían aprender los/las estudiantes en el área de las Ciencias Naturales.

La Ley de Educación Nacional (Ministerio de Educación, Ciencia y Tecnología, 2007) establece en el punto «S» del Artículo 11 el «Promover el aprendizaje de saberes científicos fundamentales para comprender y participar reflexivamente en la sociedad contemporánea». Bajo esta premisa los objetivos, metodologías y recursos puestos en juego para la enseñanza fueron variando con el tiempo, acompañando las producciones teóricas y las propuestas de enseñanza que se iban generando en los países centrales, en Latinoamérica y en nuestro propio país. En los acuerdos que se han suscripto alrededor de este tema se han involucrado ámbitos de enseñanza tanto de nivel primario como secundario, de instituciones estatales y privadas. También se han visto envueltos los dedicados a la producción de conocimientos científicos y tecnológicos (universidades, centros de investigación, etc.). Mellado (2003) señala que si existe una falta de reflexión por parte del docente sobre la naturaleza del conocimiento científico, esto hace que se 
dificulte el desarrollo de competencias científicas. Se requiere un cambio de actitud docente, de manera de enriquecer su práctica. El desarrollo de competencias científicas en los estudiantes obedece al conocimiento didáctico del contenido, lo que involucra a la disciplina, la pedagogía y la contextualización (Chona et al., 2006). Particularmente en las escuelas, los/las docentes se enfrentan a grandes carencias, que tienen que ver con la poca importancia que se le da a la enseñanza de las ciencias experimentales: hay escasa o nula cantidad y calidad de recursos para la enseñanza (libros, propuestas, materiales de laboratorio) y falta de infraestructura adecuada para actividades científicas. Sumado a esto, el tiempo de clases en los primeros años de la educación es insuficiente, por lo que se abocan a la enseñanza de la lectoescritura y las matemáticas (lo que se podría subsanar en parte, teniendo cargos para personal de apoyo en actividades experimentales). Más allá de la estructura de las instituciones, es conocido que existen debilidades en la cantidad y calidad de ofertas de formación continua de los docentes en ejercicio.

Si nos centramos particularmente en la provincia de Santa Fe, el Diseño Curricular Jurisdiccional del Profesorado de Educación Primaria en vigencia, aprobado en 2009, fue pensado con el fin de unificar los fragmentados planes de estudio del profesorado de educación primaria de instituciones terciarias públicas y privadas de la provincia (Ministerio de Educación, 2009). Una consulta del documento muestra que solo se menciona una vez la palabra «laboratorio» en todo el plan de estudios, dentro de la currícula del taller «Ciencias Naturales para una cultura ciudadana», que se dicta en el primer año de la carrera. Esto conlleva a los/las docentes a no sentirse seguros en desarrollar actividades experimentales por desconocimiento e inseguridad debido a que no se formaron durante su trayectoria estudiantil. Otra consecuencia de desestimar las actividades experimentales como parte del aprendizaje en la formación de los/las futuros/as docentes, es la falta de espacios físicos habilitados a tal fin en las instituciones educativas primarias, o que aquellos lugares que fueran destinados a laboratorios sean finalmente empleados como depósitos de libros, artículos de educación física, archivos de la escuela, etc. Esta situación ha sido relevada en diferentes escuelas 
que participaron de proyectos de investigación educativos llevados a cabo por nuestro grupo de investigación.

A través del plan Ciencias Naturales para Todos, promulgado por el Ministerio de Educación de la Nación Argentina a través de la Dirección Nacional de Gestión Educativa y su Dirección de Educación Primaria (Consejo Federal de Educación, 2012), se inició un proceso de desarrollo profesional docente, priorizando un trabajo reflexivo respecto de la aplicación de la indagación, en la didáctica de las ciencias naturales. Del mismo modo, se intentó acercar la cultura científica al aula incorporando los modos de construcción en las Ciencias Naturales como objetivos centrales de la enseñanza.

El proyecto tenía por objetivo generar mejores condiciones para la enseñanza y el aprendizaje de las Ciencias Naturales según lo descripto por los Núcleos de Aprendizaje Prioritarios para el segundo ciclo de la enseñanza primaria (Ministerio de Educación, 2005), facilitar el acceso al profesorado y al estudiantado al instrumental y material bibliográfico, promover el aprendizaje a través de procedimientos científicos escolares y repensar las prácticas, dinámicas y tiempos en el aula de forma de generar mejores estrategias de trabajo. Lamentablemente, este plan no tuvo continuidad en el tiempo e incluso los materiales disponibles en línea fueron dados de baja. Sumando a esto, durante los recorridos de nuestro grupo de investigación a escuelas primarias se constató que, si bien en la mayoría de los establecimientos educativos contaban con este material, el mismo se conservaba con el empaque usado para su transporte ya que los docentes no lo usaban en sus clases. Frente a esta realidad, se decidió llevar a cabo talleres de capacitación para docentes de escuelas primarias cuyo principal objetivo fue la divulgación del conocimiento científico-tecnológico por medio de la realización de actividades prácticas, experiencias y demostraciones de fenómenos físicos, químicos y biológicos. Por otro lado, también se priorizó la difusión de estrategias, herramientas y técnicas innovadoras que permitan actualizar contenidos estructurantes de las Ciencias Naturales, poniendo en valor el laboratorio móvil.

Toda instancia de taller, al igual que cualquier proceso educativo, busca promover entre los y las participantes, un cambio conceptual, actitudinal y valorativo sobre un tema, objeto o actividad determinada. Los talleres tienen 
la particularidad de integrar, en su propia dinámica, la teoría con la práctica. De este modo, el experimento, como recurso didáctico, resulta sumamente valioso por contener per se, esa dicotomía teoría-práctica, la cual exige la participación cognitiva y física de los y las estudiantes (Rodríguez Sánchez y Vargas Ulloa, 2009).

En el presente trabajo, se describen una serie de experiencias de laboratorio llevadas a cabo junto con las y los docentes participantes, como así también sus apreciaciones y perspectivas para un trabajo a futuro.

\section{Metodología}

Se realizaron instancias de capacitación situada en dos escuelas de la provincia de Santa Fe: una ubicada en la ciudad de Reconquista -en el norte provincial- y otra localizada en Santa Fe capital. Cada instancia fue realizada con la modalidad taller y tuvo una duración de tres horas. Se trabajaron contenidos estructurantes de las disciplinas que conforman el área Ciencias Naturales: materia y sus transformaciones, estados de agregación, mezclas homogéneas y heterogéneas, métodos de fraccionamiento y métodos de separación de fases, células, seres vivos y sus características, funciones de nutrición, química de alimentos, entre otros.

En cada una de las experiencias se utilizaron sustancias de la vida cotidiana de fácil accesibilidad, resguardando la integridad física de los y las estudiantes y los y las docentes. También se propició el uso de cámaras fotográficas y celulares para la captura de microfotografías. Cabe señalar que a la mayoría de las experiencias realizadas se les asignó un nombre metafórico, dando cuenta de un uso connotativo del lenguaje.

A modo de resumen de lo antes expuesto, se presenta la Tabla 1, donde además se especifican los materiales de laboratorio utilizados, el cual fue entregado el marco del programa "Ciencias Naturales para Todos".

El detalle de cada una de las experiencias antes descriptas puede ser consultado en el libro El Laboratorio en el Aula (Reyes y Granados, 2017). Al finalizar las instancias de capacitación se suministró a cada docente una encuesta con la finalidad de conocer aspectos vinculados a las actividades 


\section{Tabla 1.}

Resumen de las experiencias presentadas en el taller, considerando los contenidos curriculares y el material disponible en las escuelas a partir del plan "Ciencias Naturales para Todos".

\begin{tabular}{|c|c|c|}
\hline Material Disponible & Contenido Curricular Trabajado & Nombre y/o Descripción de la Experiencia \\
\hline $\begin{array}{l}\text { Microscopios, porta y } \\
\text { cubreobjetos. } \\
\text { Pipeta Pasteur. } \\
\text { Bisturí }\end{array}$ & $\begin{array}{l}\text { Seres Vivos - Células. } \\
\text { Alimentación y Nutrición. }\end{array}$ & $\begin{array}{l}\text { Observación de células vegetales y animales. } \\
\text { Observación de gránulos de almidón en diferentes } \\
\text { muestras de alimentos. }\end{array}$ \\
\hline $\begin{array}{l}\text { Papel de filtro. } \\
\text { Vasos de Precipitados. } \\
\text { Varilla. } \\
\text { Capilares. }\end{array}$ & $\begin{array}{l}\text { Mezclas homogéneas y métodos de } \\
\text { fraccionamiento. }\end{array}$ & $\begin{array}{l}\text { Separación de los componentes de una tinta a través } \\
\text { de una cromatografía en papel: resolución de una } \\
\text { situación problemática. }\end{array}$ \\
\hline $\begin{array}{l}\text { Tubos de ensayo. } \\
\text { Gradilla } \\
\text { Vasos de Precipitado. }\end{array}$ & $\begin{array}{l}\text { Mezclas heterogéneas. } \\
\text { Densidad y Miscibilidad. }\end{array}$ & $\begin{array}{l}\text { "Semáforo líquido", "Arco iris de Azúcar" y "Torre de } \\
\text { Colores": realización de mezclas heterogéneas } \\
\text { utilizando soluciones de diferentes densidades. }\end{array}$ \\
\hline $\begin{array}{l}\text { Placas de Petri. } \\
\text { Vasos de Precipitados. } \\
\text { Pipetas Pasteur. }\end{array}$ & $\begin{array}{l}\text { Alimentación y Nutrición. } \\
\text { Reacciones Químicas. }\end{array}$ & $\begin{array}{l}\text { "Detective Alimentario" Realización de experiencias } \\
\text { para la determinación cualitativa de proteínas y } \\
\text { almidón en diferentes muestras alimentarias. }\end{array}$ \\
\hline $\begin{array}{l}\text { Erlenmeyer. } \\
\text { Probeta. } \\
\text { Cucharita. }\end{array}$ & Reacciones Químicas. & $\begin{array}{l}\text { "La Naranja Infladora": obtención de dióxido de } \\
\text { carbono a partir de la reacción entre el ácido cítrico y } \\
\text { el bicarbonato de sodio. }\end{array}$ \\
\hline
\end{tabular}

experimentales que realizan en el aula, las dificultades que encuentran y sus apreciaciones sobre el taller. Las encuestas fueron suministradas en dos formatos: escrita y en Google Formularios, para docentes que no pudieran completar la encuesta finalizado el taller (link de acceso es https://forms.gle/ ihvkxrWa3m38zdW58). En la Tabla 2 se detallan las preguntas realizadas.

\section{Resultados}

De los cuarenta y cinco docentes que participaron en los talleres, dieciocho respondieron a la encuesta. Cabe destacar que se les envió por correo electrónico a todos/as los/as participantes un link para completarla de forma online con recordatorios semanales durante dos meses. En cuanto al 


\section{Tabla 2.}

Preguntas realizadas a los docentes a manera de evaluación del taller.

\begin{tabular}{|c|c|}
\hline \multirow[t]{2}{*}{ Datos Personales } & Dirección de mail \\
\hline & Asignatura que dicta \\
\hline \multirow[t]{4}{*}{ Sobre la experimentación en clases } & ¿Solía emplear experiencias prácticas en sus clases? Si, No, A veces \\
\hline & ¿Cree que las empleará ahora? Si, No, A veces \\
\hline & ¿Cuáles cree que son las dificultades que puede tener su aplicación? \\
\hline & ¿Cuáles tópicos cree que podría abordar con ellas? \\
\hline \multirow[t]{2}{*}{ Evaluación del Taller } & ¿Cómo evaluaría el Taller? ¿Qué incorporaría o sacaría? \\
\hline & Si lo desea, puede dejar un comentario o sugerencia. \\
\hline
\end{tabular}

análisis de las encuestas, encontramos que ocho de los dieciocho docentes que respondieron ya realizaban con frecuencia algún tipo de experiencia en sus aulas, cinco solo lo realizaban circunstancialmente, mientras que cinco no lo hacían. Al preguntarles si a partir del dictado del taller consideran que comenzarían a realizar más trabajo de laboratorio solo dos personas contestaron que no, pero debido a que no encontraban relación con su espacio curricular o a que ya no ejercerían como docentes por las recientes titularizaciones de cargos directivos. Finalmente, cuando se les preguntó por posibles dificultades que encuentran al momento de aplicar experiencias en el aula la mitad de las respuestas revelaron la ausencia de dificultad para llevar a cabo este tipo de experiencias. Dos docentes respondieron que necesitan tener una mayor formación en el uso de material de laboratorio y conceptos asociados. Un mismo número manifestó que la posibilidad de la 
rotura de los materiales de laboratorio, es una limitante a la hora de llevar a cabo las experiencias. También comentaron que tener muchos alumnos, espacio de laboratorio reducido o la edad del estudiantado, eran factores que los hacían evitar este tipo de instancia de aprendizaje. Finalmente, algunos participantes comentaron que la falta de tiempo para llegar a dar todo lo que la currícula dictamina hace que no realicen experiencias en el laboratorio. Respuestas similares fueron obtenidas por García-Ruiz y Orozco-Sánchez (2008), donde a través de encuestas a docentes de nivel primario, los mismos reconocieron que hacían uso de cuestionarios, resúmenes, exposiciones orales de los temas con la ayuda de esquemas o ilustraciones y lectura del libro de texto, pero en muy pocas oportunidades se realizaban en actividades experimentales o prácticas, argumentando que no cuentan con la formación adecuada, como así tampoco con el material correspondiente.

Cuando se les preguntó sobre los tópicos con los que trabajarían en el laboratorio, obtuvimos respuestas similares entre docentes de la misma institución. En una de las escuelas, plantearon que emplearían experiencias para trabajar el método científico o como un recurso disparador para despertar curiosidad en sus estudiantes; mientras que, en la otra escuela, se limitaron a repetir los contenidos del taller en cada una de sus respuestas. La mayoría de los y las docentes lo calificó como «Excelente», «Interesante» o «Muy bueno», remarcando la utilidad de este tipo de instancias de formación. Solo una docente comentó que le parecía «bueno» pero debido a que el taller se dictó a fines de año y consideraba que este tipo de formación solo debe darse al inicio del ciclo lectivo.

Resultados similares, en cuanto a la buena recepción de estas prácticas, fueron obtenidos por García-Ruiz y Orozco-Sánchez (2008), Zabalegui y Santos Duarte (2014) y Furlani et al.,(2016), donde equipos de docentes-investigadores realizaron talleres en diferentes instituciones educativas con el objetivo de acercar contenidos estructurantes de las Ciencias Naturales a través de la experimentación.

Finalmente, cuando preguntamos por posibles sugerencias y comentarios, los y las participantes plantearon la necesidad de seguir realizando talleres con cierta periodicidad, con temáticas diversas, y la edición de algún material escrito. Este último aspecto fue Ilamativo, puesto a que el plan «Ciencias 
Naturales para todos» incluía material impreso. Cuando decidimos explorar el sitio web del Ministerio de Educación de la República Argentina encontramos que el enlace hacia los libros que podían usar los y las docentes con experiencias para aprovechar el material de laboratorio brindado, ya no existía.

\section{Consideraciones finales}

Teniendo en cuenta los resultados obtenidos, consideramos que el dictado de los talleres fue altamente satisfactorio. Sin embargo, la sostenibilidad en el tiempo de estas propuestas de capacitación, requiere de una planificación estratégica y del pronunciamiento de políticas públicas acordes, con el propósito de privilegiar los procesos cognitivos para el desarrollo de diferentes habilidades. En este sentido y tomando palabras de Harlen (2008), estamos convencidos que enseñar a pensar científicamente, permite a los estudiantes acceder a modos de razonamiento basados en la evidencia, brindando la satisfacción de encontrar respuestas por uno mismo a nuevos interrogantes, permitiendo flexibilidad en el pensamiento y fundamentalmente, promoviendo en el estudiantado la capacidad y el deseo de seguir aprendiendo.

\section{Agradecimientos}

El equipo de investigación agradece la financiación de la Agencia Nacional de Promoción Científica y Tecnológica (PICT № 2016 - 0594). Agradecemos además a las escuelas participantes, sus equipos directivos y docentes por la participación de los talleres y la respuesta de las encuestas.

Esta investigación se llevó adelante en el marco de la tesis doctoral «Propuestas Innovadoras en la Enseñanza de las Ciencias Experimentales: el rol de las preguntas-problematizadoras del docente y el alumno en distintos niveles del Sistema Educativo» (Expte. N933348 - 18; Res. N³94). 


\section{Referencias bibliográficas}

Chona, G., Arteta J., Fonseca, G., Ibáñez, X., Martínez, S., Pedraza, M., \& Gutiérrez, M. (2006). ¿Qué competencias científicas desarrollamos en el aula? Revista TES Tecné, Episteme y Didaxis, (20), 62-79.

Consejo Federal de Educación (2012). Resolución CFE $N^{\circ} 188 / 12$. Argentina. Disponible en http://nuestraescuela.educacion.gov.ar/bancoderecursosnivelsuperior/ seccion 1/Resoluci\%C3\%B3n\%20188-12-con\%20 anexos.pdf. Última revisión 08/07/2020.

Furlani, M., Palma, S., Rebechi, S. y Salsi, S. (2016). Apertura al conocimiento en Ciencias Naturales a través de la experimentación. Revista $+E,(6), 352-359$.

García-Ruiz, M. y Orozco-Sánchez, L. (2008). Orientando un cambio de actitud hacia las Ciencias Naturales y su enseñanza en Profesores de Educación Primaria. Revista Electrónica de Enseñanza de las Ciencias, 7 (3), 539-568.

Harlen, W. (2008). Teaching, learning and assessing science K-12. Londres: SAGE Publications.

Mellado, V. (2003). Cambio didáctico del profesorado de ciencias experimentales y filosofía de la ciencia. Enseñanza de las ciencias, 21 (3), 343-358.

Ministerio de Educación, Ciencia y Tecnología de la República Argentina. (2005). Núcleos de Aprendizaje Prioritario para el $2^{\circ}$ Ciclo de la EGB/Nivel Primario. Argentina. Disponible en: http://www.bnm.me.gov.ar/ giga1/documentos/EL000972.pdf. Última revisión: 08/07/2020.
Ministerio de Educación, Ciencia y Tecnología de la República Argentina. (2007). Ley Nacional de Educación № 26.206 «Hacia una educación de calidad para una sociedad más justa». Argentina. Disponible en http:// www.bnm.me.gov.ar/giga1/documentos/EL002609. pdf. Última revisión 08/07/2020.

Ministerio de Educación de la provincia de Santa Fe. (2009). Resolución $N^{\circ} 528$, Expediente $N^{\circ} 00401$ 0187301-7. Santa Fe, Argentina. Disponible en: https://www.santafe.gov.ar/index.php/educacion/content/ download/122509/606630/file/528-09\%20Primario. pdf. Última revisión: 08/07/2020.

Reyes, M.S. y Granados, D.I. (2017). El laboratorio en el aula. Santa Fe: Ediciones UNL.

Rodríguez Sánchez, K. y Vargas Ulloa, K. (2009). Análisis del experimento como recurso didáctico en talleres de ciencias: el caso del Museo de los Niños de Costa Rica. Actualidades Investigativas en Educación, 9 (1), 1-20

Zabalegui, M. F. y Santos Duarte, C. (2014). Espacios alternativos de enseñanza de las ciencias. Revista de Educación en Biología, 17 (2), 111-117. 